\title{
MISSING LEVELS AND LINES OF ASTROPHYSICAL IMPORTANCE
}

\author{
SVENERIC JOHANSSON \\ (Department of Physics, \\ University of Lund, \\ Lund, Sweden)
}

\author{
and
}

CHARLES R. COWLEY

(University of Michigan

and Dominion Astrophysical observatory)

The purpose of this paper is to make a brief survey of the knowledge of iron group elements as concerns energy levels and transition arrays in neutral and singly ionized atoms and to give rough predictions of missing levels and lines. The amount of data that have been published for these elements after the edition of the Multiplet Tables are considerable. For detailed information about sources of new data and compilations the reader is referred to the Report from Commission 14 in the Transactions of IAU. In this paper we will focus on still missing energy levels and associated missing transitions, which have to be considered in the construction of synthetic spectra.

The structure of the iron group elements is complex not only because of the filling of the $3 \mathrm{~d}$ shell but also because of the competition in binding energy between the $3 \mathrm{~d}$ - and the $4 \mathrm{~s}$ electron. For a given number of valence electrons, $k$, we have to consider three different low configurations in the complex $(3 \mathrm{~d}+4 \mathrm{~s}){ }^{\mathrm{k}}$ having about the same energy in neutral and singly ionized atoms. In neutral atoms the $3 \mathrm{~d}^{\mathrm{k}-2} 4 \mathrm{~s}^{2}$ configuration generally occupies the ground state, while the $3 \mathrm{~d}^{\mathrm{k}}$ configuration appears at rather high excitation energy. In the singly ionized atoms all three configurations have about the same energy, giving the most pronounced complexity.

The importance of these three configurations is reflected in the observed spectra of the iron group elements, since the lower level in all strong transitions generally belongs to one of them. For an analysis of a stellar spectrum in absorption the knowledge of more or less all levels in the complex $(3 d+4 s)^{k}$ is therefore nessecary. Detailed tables of all spectroscopic terms - known as well as unknown - in these configurations of first and second spectra are displayed on posters. Here we give the data in a condensed form in Table 1, which is aimed to illustrate the present knowledge of these low configurations. The first two columns give the ionization potential (I.P.) and the total number of spectroscopic terms in the $(3 d+4 s)^{k}$ complex. The third column yives the number of known terms as a fraction of the total number and in the last column the excitation potential (E.P.) of the lowest unknown term is expressed as a fraction of the I.P.

The by far most prominent Iines in the spectra of iron group elements are assigned to $3 \mathrm{~d}-4 \mathrm{p}$ or $4 \mathrm{~s}-4 \mathrm{p}$ transitions. For the neutral atoms these 1 ines fall well above $3000 \AA$ while they in singly ionized atoms build up the heavy resonance region below $3000 \AA$. In order to get an idea of in which spectral region different transitions occur we have plotted the position of various configurations as a function of element in Fig 1. As a zero level we have chosen the lowest term of the $3 \mathrm{~d}^{\mathrm{k}} 4 \mathrm{~s}$ configuration. Different curves then represent $3 \mathrm{~d} \mathrm{kl}$ configurations in the first (faint lines and dots) and second (bold 1 ines and crosses) 99 


\section{Table 1}

\begin{tabular}{|c|c|c|c|c|}
\hline Spectrum & I.P. (eV) & $\begin{array}{l}\text { Number of } \\
\text { Total }\end{array}$ & $\begin{array}{l}\text { terms } \\
\text { known }\end{array}$ & E.P. \\
\hline Sc I & 6.56 & 16 & 1.00 & - \\
\hline Sc II & 12.89 & 8 & 1.00 & - \\
\hline $\mathrm{Ti} I$ & 6.83 & 37 & 0.57 & 0.50 \\
\hline $\mathrm{Ti}$ II & 13.63 & 16 & 1.00 & - \\
\hline V I & 6.74 & 48 & 0.42 & 0.39 \\
\hline$V I I$ & 14.2 & 37 & 0.95 & 0.52 \\
\hline $\mathrm{Cr} \mathrm{I}$ & 6.76 & 64 & 0.44 & 0.62 \\
\hline Cr II & 16.49 & 48 & 0.88 & 0.48 \\
\hline Mn I & 7.43 & 48 & 0.35 & 0.65 \\
\hline Mn II & 15.64 & 64 & 0.64 & 0.47 \\
\hline $\mathrm{Fe} \mathrm{I}$ & 7.90 & 48 & 0.65 & 0.52 \\
\hline $\mathrm{Fe}$ II & 16.18 & 48 & 0.79 & 0.53 \\
\hline Co I & 7.86 & 16 & 0.81 & 0.51 \\
\hline Co II & 17.05 & 37 & 0.51 & 0.31 \\
\hline $\mathrm{Ni} \mathrm{I}$ & 7.63 & 7 & 0.86 & 0.80 \\
\hline $\mathrm{Ni} I I$ & 18.15 & 16 & 0.88 & 0.42 \\
\hline
\end{tabular}

spectra as a function of $k$ in an iso-ionic comparison of the excitation energies. A configuration is represented by that particular level which is built on the lowest parent term and has the highest J-value. As we see in Fig 1 we get smooth curves and it will be easy to predict the position of missing configurations by extrapolation (e.g. 5p in Ti I). If we now take the energy difference between two curves in Fig 1 , which represent configurations of different parity in the same spectrum, we get the wavenumber of the strongest transition in that particular transition array. These differences are plotted in Fig. 2 and the curves give an idea of where the strongest transitions occur in different spectra. Most of these lines are known (circles for predicted lines) since they represent transitions between levels built on the lowest parent term and thus have the biggest chance to show up in the spectrum. However, from Table 1 we can understand that there are still missing lines between levels built on higher parent terms. Generally, we can expect these transitions to occur at roughly the same wavelength as the corresponding electronic transition in Fig 2, since the excitation energy of the high parent term shift the two curves in Fig 1 by the same value. Since there are more than one term associated to one particular parent term we can expect the wavelength region covered by an electronic transition to be extended by about 2-300 $\AA$ around the plotted wavelength.

In Fig 2 we can see that the $4 \mathrm{p}-5 \mathrm{~s}$ and $4 \mathrm{p}-4 \mathrm{~d}$ transitions in the second spectra fall in the same wavelength region as the $4 \mathrm{~s}-4 \mathrm{p}$ lines, which of course complicates the analysis of IUE spectra. (From present studies of $F e$ II in this region it is quite clear that a great number of strong lines remain unidentified.) The next lowest groups of lines extend below $2000 \AA$ and refer to the $4 p-5 d$ and $4 p-6 s$ transition in second spectra. The probability for them to occur in stellar spectra can be discussed in terms of branching ratios for different members in 


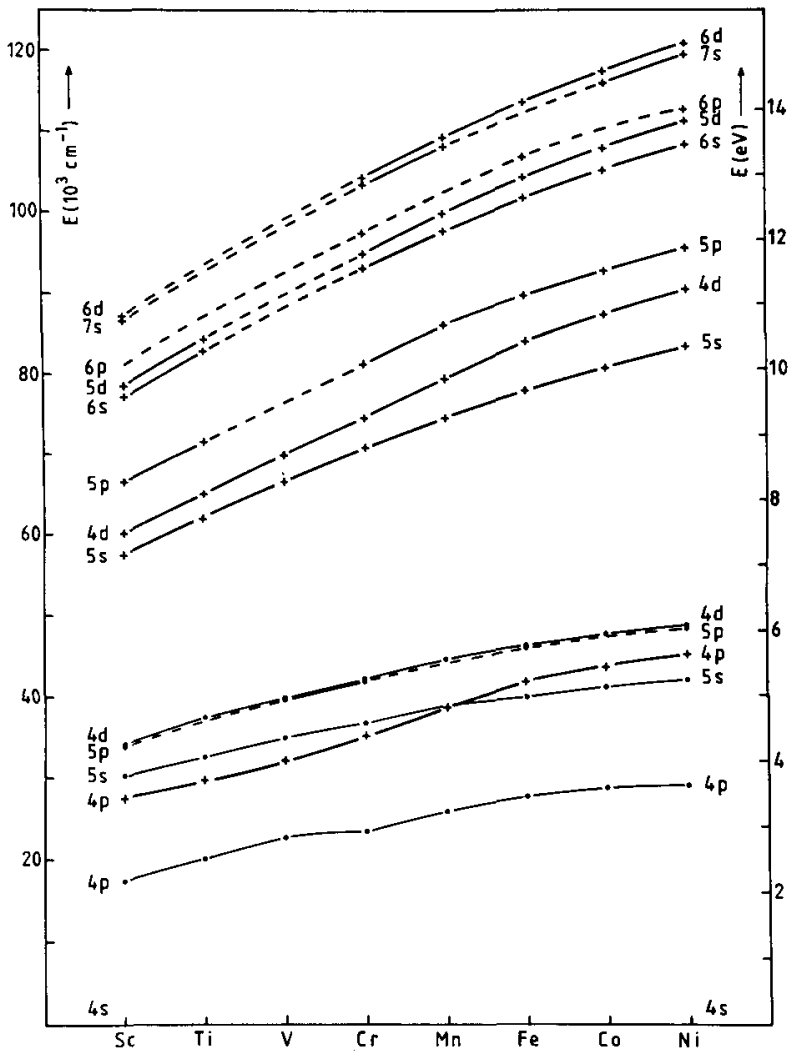

Fig..1 Excitation energies for $3 d^{k} 1$ configurations in first (dots) and second (crosses) spectra of the iron group elements

the $s$ - and d-series. The f-values for the $4 p-5 s$ and $4 p-4 d$ lines are of the same order of magnitude ( 0.1 and 0.6 respectively) but roughly a factor of ten smaller for the $4 p-6 s$ and $4 p-5 d$ transitions.

In the wavelength region below $2500 \AA$ we also expect the $4 s-n p(n>5)$ transitions of the first spectra to occur. Laboratory measurements of the p-series have been done (in absorption) in a number of spectra. From oscillator strengths in Cr I (Huber et al 1975) we can get an idea of the scaling of the f-values in the p-series: $4 s-4 p f=0.11$, $4 s-5 p \quad f=0.014,4 s-6 p f=0.0022$ i.e. roughly a factor of ten when going up in a Rydberg series.

In this brief review of missing 1 ines we have only considered transitions within one of the three possible systems of configurations, namely the $3 \mathrm{~d}^{\mathrm{k}} \mathrm{nl}$ system. As we pointed out above the $3 \mathrm{~d}^{\mathrm{k}}-3 \mathrm{~d}^{\mathrm{k}} \mathrm{I} \mathrm{np}$ transitions are very dominating in second spectra and give strong lines in the IUE region. They can not be represented in such a smooth curve in the diagram as the plotted transitions. In general we can 


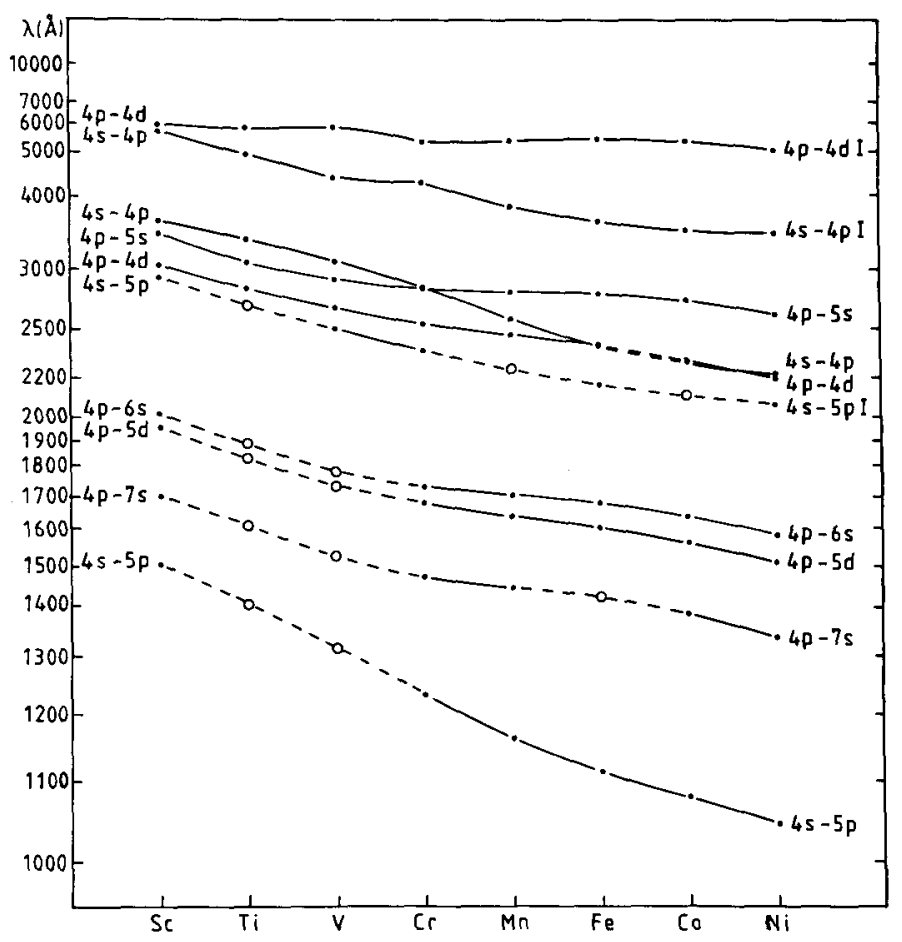

Fig 2. Representative wavelengths for some transitions within the $3 d \mathrm{k} l$ system in the first and second spectra of the iron group elements.

state that all possible LS transitions between $3 \mathrm{~d}^{\mathrm{k}}$ and $3 \mathrm{~d}^{\mathrm{k}-1} 4 \mathrm{p}$ are strong. More or less all $3 \mathrm{~d}^{\mathrm{k}}$ levels are known in second spectra and since that is also the case for the $3 \mathrm{~d}^{\mathrm{k}-1} 4 \mathrm{p}$ configuration nearly all prominent $l$ ines are known.

As was said in the introduction the $3 \mathrm{~d}^{\mathrm{k}-2} 4 \mathrm{~s}^{2}$ configurations play the key role in the neutral atoms and the associated system of configurations $3 \mathrm{~d}^{\mathrm{k}-2} 4 \mathrm{snl}$ account for the major part of the known levels in these spectra. The lowest excited configuration is thus $3 d^{k-2} 4 \mathrm{~s} 4 \mathrm{p}$ which generally deexcites in the resonance lines. In order to get smooth curves for this type of configurations the levels must be plotted versus the grand parent terms of $3 \mathrm{~d}^{\mathrm{k}-2}$ The $3 \mathrm{~d}^{\mathrm{k}-2} \mathrm{nl}$ configurations are fairly well known in neutral atoms but poorly known in singly ionized atoms.

\section{Reference}

Huber,M.C.E., Sandeman,R.J., and Tubbs,E.F.1975, Proc R Soc Lond A342,431. 


\title{
DISCUSSION (Cowley and Johansson)
}

ADELMAN: Co-adding of spectra is an important technique for improving the signal-to-noise ratio for non-variable stars. Those who are interested should look at my paper on co-adding of Dominion Astrophysical observatory spectrograms, which is a poster paper at this Colloquium. I want to make two comments about this technique. First, stars which do not have sharp lines do not have to be observed with high resolution. One can do better by observing them with lower resolution and co-adding the spectra. Second, it is a waste of valuable telescope time to obtain inferior data if better data can be obtained by requesting it from another observer.

Jeff Fuhr at the US National Bureau of Standards has given me some data on gf-values for $\mathrm{Fe} I, \mathrm{Fe}$ II, Ti II, and Co II, and has authorized me to distribute it here. There are many NBS publications available which would interest everyone working on atomic spectra; Drs. W. Martin, J. Fuhr, and R. Zalubas are glad to give these free to anyone who requests them. They only ask that you write to them on your institution's official stationery when you request it. Their address is:

\author{
National Bureau of Standards \\ Physics Building - Room A267 \\ Gaithersburg, MD 20899 USA
}

HUBENÝ: I would like to show you an example which illustrates the problem of misidentification in IUE spectra. Hubený, Štefl \& Harmanec (Bull. Astr. Inst. Czech., 36, p. 214, 1985) show that blends of numerous $F e$ III lines produce two fictitious lines, closely simulating the strong resonance lines of $C$ IV, for moderately and rapidly rotating B3 to B8 stars. Even at lower temperatures this can be a problem, because one gets what looks like C IV lines, but shifted about $100 \mathrm{~km} / \mathrm{s}$. This effect is seen in many Be stars! Here [shows slide] the fictitious blend is due to $\mathrm{Fe} I I$.

SEVERNY: You did not mention the possibility of astrophysical determination of $g f$-values using stars with known chemical composition. We have had problems analysing UV spectra obtained with Astron, because Kurucz and Peytremann gf-values are sometimes wrong by factors of $10^{3}$ to $10^{4}$. What is your opinion of this method?

COWLEY: This is certainly an important method. However, if there are additional unknown lines which are blends, we can make many errors with this method, as $\mathrm{Dr}$. Hubeny has just pointed out. Like all things, it has its good and bad parts. In general I prefer to use the NBS critically evaluated gf-values when they are available. of course, there are many lines for which gf-values are not known, and we have to use the astrophysical method.

JOHANSSON: I'm glad to see that the data we are producing at Lund is of good help for your spectral analysis of stars. We have worked on Fe II since 1974, and this work is continuing. We are analysing the entire IUE region, in collaboration with Dr. Baschek in Heidelberg. We hope to produce a new multiplet table for $\mathrm{Fe} I I$. We are also working on other 103

C. R. Cowley et al (eds.), Upper Main Sequence Stars with Anomalous Abundances, 103-104.

(C) 1986 by D. Reidel Publishing Company. 
iron-group elements. If you want some laboratory line lists, we can send these to you. In some wavelength regions there is a severe lack of atomic data for the iron-group.

COWLEY: I have one small additional comment on the Kurucz (1981) Fe II calculations. In the region discussed in my paper, the strongest contributions came from transitions to certain odd upper levels which had not been properly described quantum-mechanically in his work. These oscillator strengths are almost certainly greatly overestimated, and contribute roughly $10 \%$ to the general absorption there. Undoubtedly, that absorption belongs someplace, but not precisely at the wavelengths where it was calculated.

ARTRU: Even for Si II, which is considered to be a relatively wellknown atomic spectrum, about $30 \%$ of the multiplets (many of them strong) have no known gf-values. About $25 \%$ are missing in the Kurucz \& Peytremann list, because in Si II there are many doubly excited levels which have mixed configuration effects. So, even for a 'simple' ion like Si II there are problems.

\section{DISCUSSION (Johansson and Cowley)}

HUBENÝ: From the practical point of view, it would be nice to know that lines originating between newly found energy levels are generally weaker than previously known lines from well-established levels. Is there such a hope at present, or in the near future?

JOHANSSON: We know already that a great number of $\mathrm{Fe}$ II lines, which are not available in the literature, have $\log \mathrm{gf} \approx-1$ and lower excitation potentials around $7-8 \mathrm{eV}$. They appear as strong absorption features in the IUE region. For $\mathrm{Cr}$ II, no lines below 1780 \& are available in existing compilations. We now know of about 1000 new lines in this region with the strongest lines around $1430 \AA$. The gf values for these lines are certainly not more than one order of magnitude less than the $\mathrm{gf}$ values for the resonance lines. The conclusion is that a lot of predicted lines will have intensities comparable to those of 'well-established' lines. 


\title{
CHARACTERISTIC ABSORPTION FEATURES IN THE SPECTRA OF AP-SI STARS BETWEEN 1250 AND $1850 \AA$.
}

\author{
Marie-Christine ARTRU \\ Département d'Astrophysique Fondamentale \\ Observatoire de Meudon \\ F-92195 MEUDON PRINCIPAL CEDEX. France

\begin{abstract}
Thierry LANZ
institut d'Astronomie de I'Université de Lausanne and Observatoire de Genéve.
\end{abstract} \\ $\mathrm{CH} 1290$ CHAVANNES-DES-BOIS. Switzerland
}

\begin{abstract}
On the basis of IUE data. the specific absorption features in the spectra of Ap-Si Stars are displayed in the 1250-1850 $\AA$ range and identified when possible. The contribution of the Si II multiplets is calculated in LTE for a typical overabundance $\times 10^{2}$ of silicon. It accounts for only a small part of the observed absorption. Commun unidentified structures. smaller than the $1400 \AA$ depression are pointed out.
\end{abstract}

\section{INTRODUCTION}

The broad absorption features observed at $1400 \AA$ in the spectra of magnetic Ap stars is well correlated to the silicon overabundance (Jamar et al. 1978). It is interpretated as autoionization of Si II (Artru et al. 1981) but the precise identification cannot be established without new laboratory data on the highly excited levels of $\mathrm{Si}^{+}$. Our purpose is to derive from IUE spectra a refined description of all the absorption features that appears specific of Ap-Si stars in the spectral range 1250-1850 $\AA$.

More than 150 different stars. classified as Ap or Bp. have been observed by the IUE satellite. From these archives. we have selected a set of sillicon stars with effective temperatures in the $10000-14000 \mathrm{~K}$ range and large absorption features at $1400 \AA$. We present here preliminary results obtained for the limited data sample described in table 1.

\section{DESCRIPTION OF THE AP-SI SPECIFIC ABSORPTION}

To exhibit the specific absorptions in the silicon stars atmospheres. we display "relative" spectra by plotting the quantity $\log \left(F_{\lambda}(p) / F_{\lambda}(n)\right)$ versus the wavelength $\lambda: F_{\lambda}(p)$ is the observed spectral flux of the peculiar star and $F_{\lambda}(n)$ the same for a normal comparison star of similar effcctive temperature. We use calibrated net spectra. either directly obtained from the IUE low-resolution images. Or derived from highresolution images after degrading the resolution.

Such "relative" spectra are given in fig. 1. The curves a, b and c correspond to the three silicon stars of table 1 and show the striking similarity of their absorption features. For comparison the curve od relates to each other the two normal stars (HD 87901 and HD 17081).

\section{5}

C. R. Cowley et al (eds.), Upper Main Sequence Stars with Anomalous Abundances, 105-108.

(C) 1986 by D. Reidel Publishing Company. 


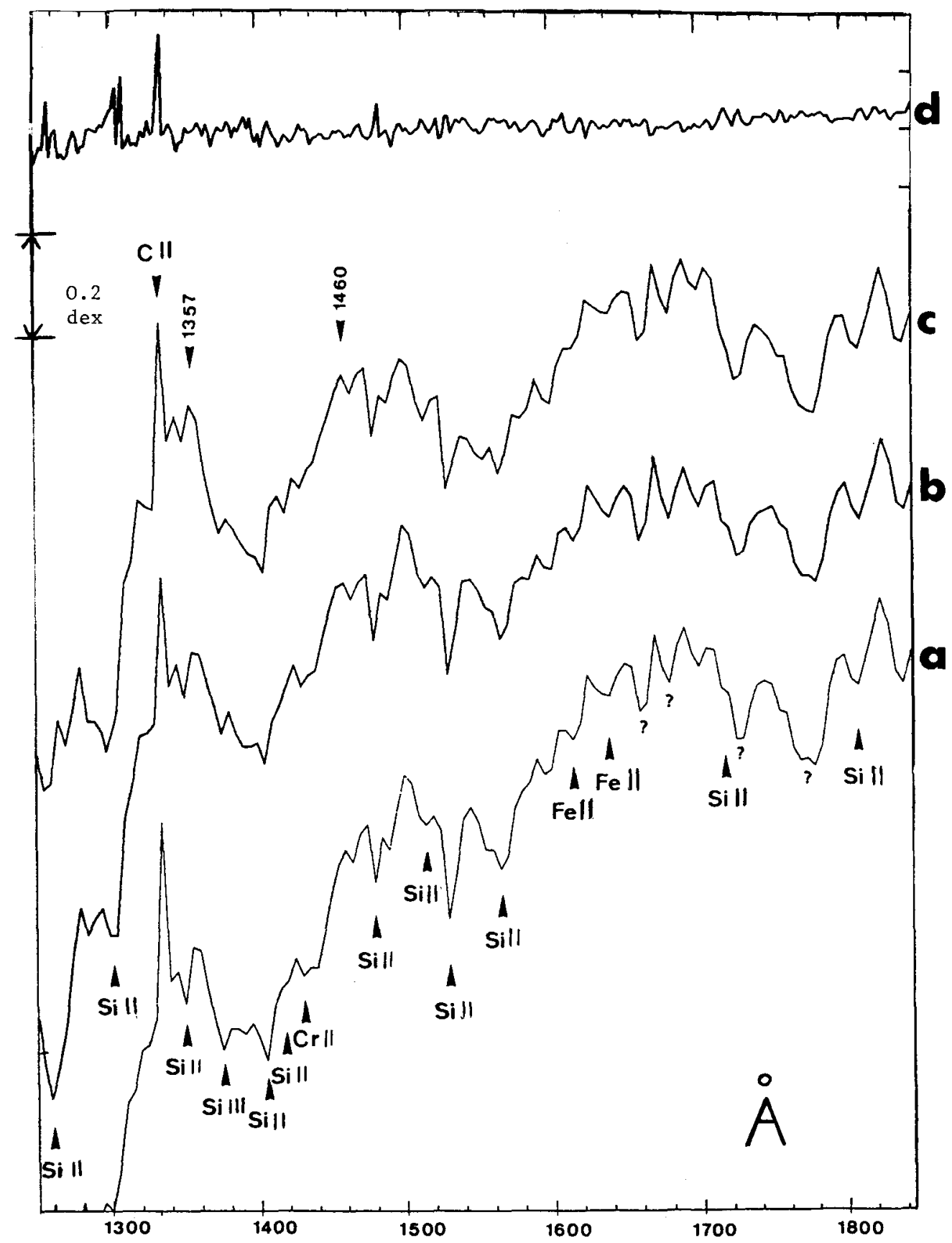

Fig. 1 - Relative spectra of three Ap-Si stars (a, b, c) and one normal star (d). The logarithm of the flux ratio is plotted for HD27309/HD17081 (a), HD25267 / HD17081 (b), HD34452/HD17081 (c) and HD87901/HD17081 (d). 


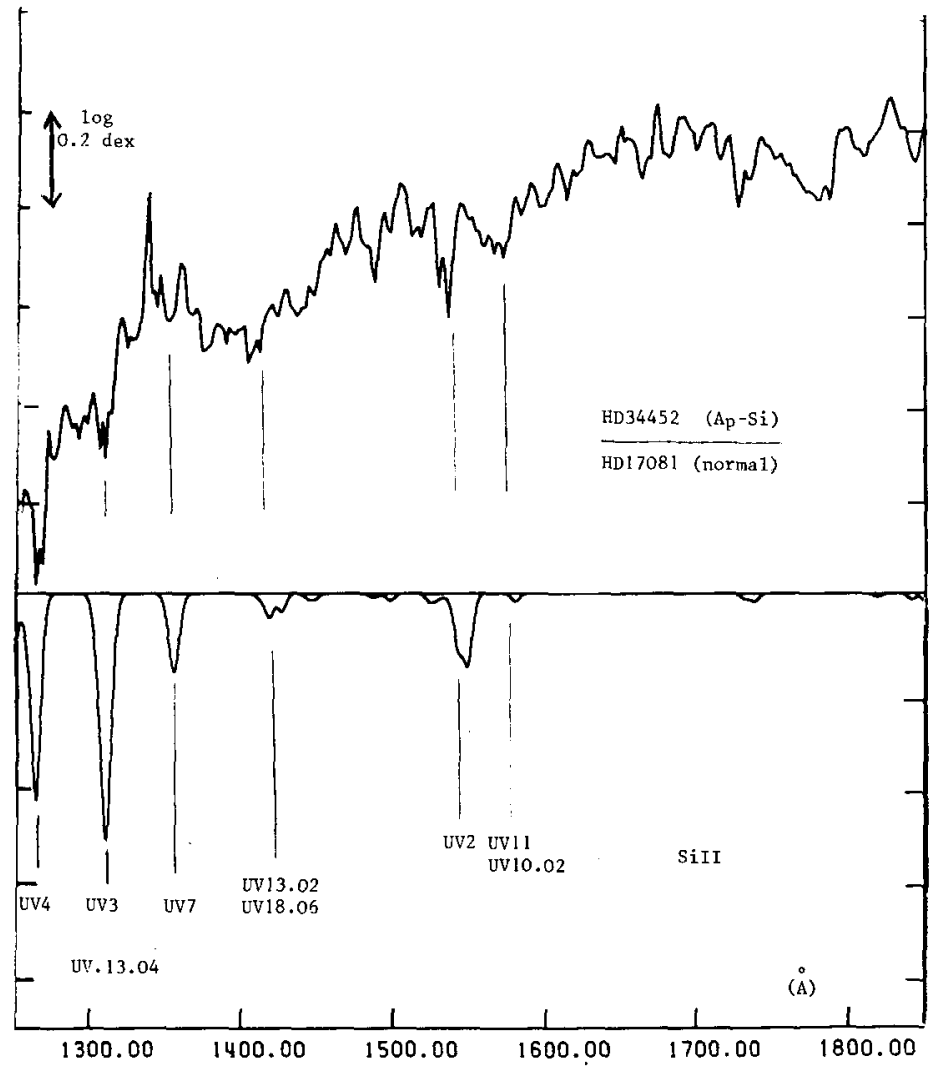

Fig. 2 - LTE synthetic spectrum of $\mathrm{Si}$ II multiplets for a silicon abundance of
$100 \times$ (lower curve) and observed "relative" spectrum of HD34452 (upper curve) (the resolution is about $6 \mathrm{~A}$ ).

Table 1 - Sample of Ap-Si stars and IUE observations

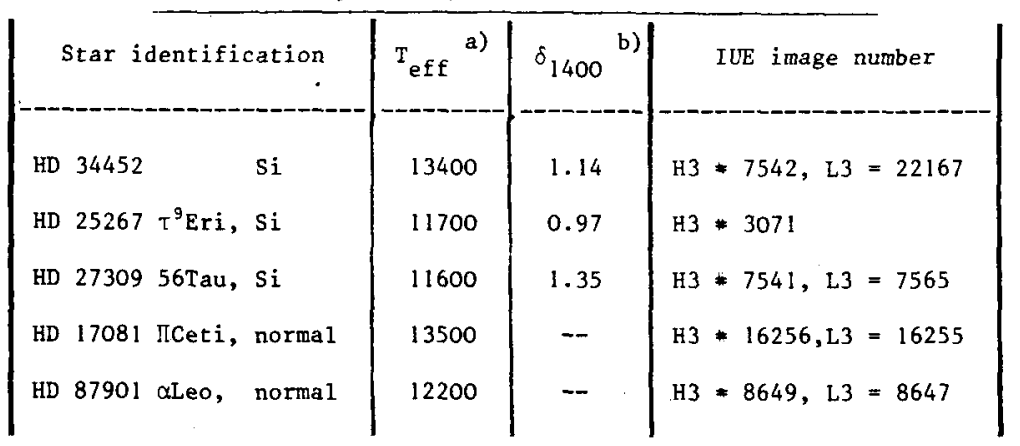

a) The effective temperature is estimated fromphotometric indexes (Lanz, 1985).

b) The $\delta_{1400}$ index measures the enhancement of the $1400 \AA$ depression (Jamar et al, 1978). 
Many of the sharper absorption lines, in the "relative" Ap -Si star spectra (fig. 1-a, b and c) are identified as strong multiplets of overabundant ions. mainly Si II and Fe II. The C II multiplet at $1335 \AA$ appears as a peak. suggesting an underabundance of carbon. A similar weaker feature also appear for the comparison star HD 87901 (flg. 1-d).

The large $1400 \AA$ feature has a similar shape for the three sillcon stars and occurs between the same limits. 1357 and $1460 \AA$. Other smaller wide depressions are observed similarly for the three SI stars. around $1550 \AA$ and $1750 \AA$. Their identification. possibly by autolonization of an overabundant ion is still an open question.

\section{THE ABSORPTION SPECTRUM OF SI $\|$}

The strongest lines identified on the observed curves of fig. 1 are due to Si II multiplets (Moore. 1965) : for instance. UV4 at $1264 \AA$. UV 13.02 and UV13.03 at $1405-1410 \AA$. UV12 and UV15. O4 at $1485 \AA$ and UV2 at $1530 \AA$.

in order to evaluate the contribution of the Si II spectrum to the opacity variations observed in fig. 1. we first calculated the total effect of the "normal" lines. as known from laboratory analysis and complled by Moore (1965). A predicted LTE synthetic spectrum of SI II was computed using a blanketed model $(13000 \mathrm{~K}, \log g=4)$ from Kurucz et al (1977). The silliclum abundance was fixed at 100 times the solar one. Which is the value generally given for HD 34452. The line list was limited to the experimental lines of SI II. with the gf-values taken from the compliation of Lanz and Artru (1985). The line spectrum of Si II obtained in this calculation is shown on fig. 2. with the same resolution and scale. as for the observed curves of fig. 1. Their comparison confirms the identification of the Si II multiplets, but shows that they contribute for only a small part. to the observed opacity. The "normal" spectra of other overabundant ions should also be calculated. but are likely not to fill the missing opacity. A systematic study of their autoionization spectra would be essential.

\section{REFERENCES}

- Artru. M. -C. Jamar, C. . Petrini. D. . Praderie. F. . 1981. Astron. Astrophys. 96. 380 .

- Jamar. C. Macau-Hercot. D. Praderie. F., 1978. Astron. Astrophys. 63. 155.

- Kurucz. R. L. . Peytremann. E. . Avrett. E. H. . 1974. Blanketed Model Atmospheres for Early-type Stars (Washington. Smithsonian Institution Press).

- Lanz. T. . 1985. Astron. Astrophys. 144. 191.

- Lanz. T. Artru. M. - C. . 1985. Physica Scripta. in press.

- Moore. C. E. . 1965. Selected Tables of Atomic Spectra. NSRDS-NBS3. National Bureau of Standards. 
David S. Leckrone

Laboratory for Astronomy and Solar Physics

Goddard Space Flight Center

Greenbelt, Maryland

USA

ABSTRACT. Progress in the spectroscopic study of $\mathrm{CP}$ stars and related sharp-lined normal stars from the IUE is briefly reviewed as a preamble to a discussion of the potential for research with the Hubble space Telescope. The substantial gains in spectral resolution, signal-to-noise ratio and photometric accuracy that will be realized with the High Resolution Spectrograph on the HST will dramatically increase our ability to disentangle the complex ultraviolet spectra of these stars and to carry out accurate quantitative analyses.

\section{INTRODUCTION}

A decade ago at IAU Colloquium Number 32 in Vienna, Leckrone (1975) reviewed the properties of $\mathrm{CP}$ stars as observed from space. At that time the observational data were limited almost entirely to broad-band photometry and intermediate-band spectrophotometry in the wavelength interval $1100 \AA$ to $3000 \AA$, which revealed the flux deficiencies of the $C P$ stars in the ultraviolet, and which explained the complex photometric variations observed in the optical region as being due to variable backwarming. Since the time of that meeting a great deal of UV spectroscopic work has been carried out on $\mathrm{CP}$ stars, primarily with the International Ultraviolet Explorer (IUE). The extended lifetime of the IUE has allowed UV spectroscopy to advance from qualitative surveys to detailed quantitative abundance analyses. Results obtained by several groups have been reported in the literature for $\mathrm{B}, \mathrm{Al}, \mathrm{Si}, \mathrm{Cu}, \mathrm{Ga}, \mathrm{Sb}$, $\mathrm{Pt}, \mathrm{Hg}$, and Bi (Jacobs and Dworetsky 1981,1982; Dworetsky et al. 1984; Leckrone 1980,1981,1984; Sadakane et al. 1983,1985). In each case the information obtained from space has provided a test of the predictions of diffusion theory, has led to the identification of new anomalous species or has supported and strengthened previous ground-based work. We are only just beginning to reap the harvest of information from the IUE, as I hope to show in this discussion and we are on the threshold of a new era of quantitative accuracy, resolution and sensitivity which will be provided by the Hubble Space Telescope (HST).

109

C. R. Cowley et al (eds.), Upper Main Sequence Stars with Anomalous Abundances, 109-119.

(C) 1986 by D. Reidel Publishing Company. 


\section{COMPREHENSIVE ABUNDANCE ANALYSES WITH IUE AND OPTICAL DATA}

Adelman and Leckrone have undertaken a long-term project to establish fundamental abundances for as many species as possible, beginning with the iron peak elements, in a selected group of $\mathrm{CP}$ and normal, sharplined B and A stars. The approach is to combine high quality, composited IUE spectra (Adelman and Leckrone 1985) with high signal-to-noise optical spectra in fully self-consistent abundance analyses. Since our data encompass the wavelength range from about $1250 \AA$ to about $6000 \AA$, we can cover a larger fraction of the periodic table, a wider range of excitation and ionization states, and for many species a statistically more significant sample of lines than can be achieved from optical data alone. The ultraviolet spectra not only make available low excitation or resonance lines of many important species but also in some cases provide lines whose oscillator strengths and damping constants are more accurately determined than those of their counterparts at optical wavelengths. On the other hand all such analyses are currently limited by the high line density of ultraviolet spectra, the lack of accurate atomic data for most of the contaminate blending lines and the rather low resolution $(\lambda / \Delta \lambda \simeq 15000)$ and signal-to-noise ratio ( $<30)$ of the IUE data. We are still in the process of learning how best to ameliorate these problems in the IUE data. In the long term the observational problems will be greatly diminished by use of the High Resolution Spectrograph (HRS) on the HST.

TABLE 1

IUE ABUNDANCE PROGRAM STARS

\begin{tabular}{rlrr} 
Star & Type & Teff & V Sin \\
\cline { 2 - 2 } Leo & A2 V & 9250 & 20 \\
0 Peg & A1 V(Am?) & 9625 & 12 \\
$V$ Cap & B9.5 V & 10250 & 17 \\
134 Tau & B9 IV & 10825 & 22 \\
21 Aq1 & B8 III & 13000 & 19 \\
$\pi$ Cet & B7 V & 13150 & 18 \\
l CrB & HgMn & 11380 & 3 \\
K Cnc & HgMn & 13300 & 6 \\
HD109995 & A2(FHB) & 8100 & 17
\end{tabular}

The stars currently being analysed in the Adelman and Leckrone abundance program are listed in Table 1. They were selected both for their low values of $v$ sin $i$ and also because a substantial amount of work on their optical spectra has already been completed. IUE data for all of these stars have been obtained and the data reduction, involving the coaddition of many spectra so as to reduce both random and fixed-pattern noise, is now complete. Our intent is to continue to update the abundances derived for these stars as improved observational or atomic data become available, thus establishing and maintaining them as reference standards. 


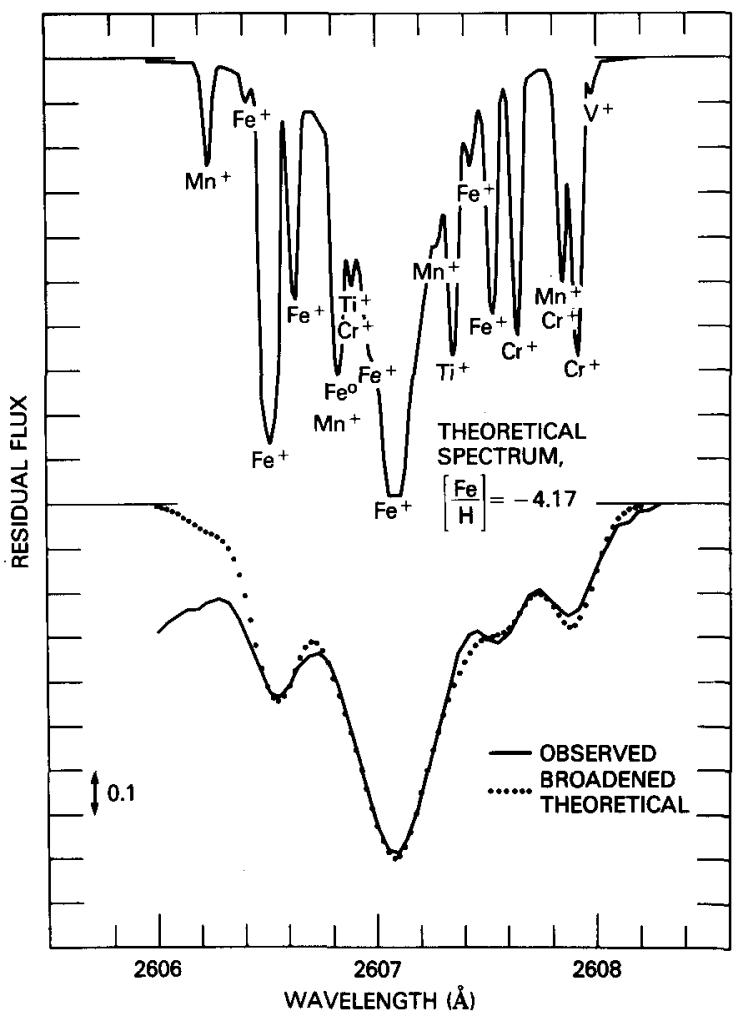

Figure 1. Spectral synthesis of the resonance line FeII $\lambda 2607$ in o Peg. Top - theoretical spectrum with $\log [\mathrm{N}(\mathrm{Fe}) / \mathrm{N}(\mathrm{H})]=-4 \cdot 17$. Bottom - IUE observation (solid curve) compared to theoretical spectrum, suitably broadened with rotation and instrument function.

The analyses have begun with iron, because its abundance is reasonably well established from the optical data alone. It thus provides a good opportunity to compare abundances obtained from both ultraviolet and optical regions for consistency. The top section of Figure 1 illustrates the computed synthetic spectrum of the resonance line, FeII(1) $\lambda 2607.086$, in o Pegasi. Note the large number of blending lines. The line lists and $\log$ gf values of Kurucz and Peytremann (1975) and Kurucz (1981) were used initially for these blending lines. But it is well known that these data contain systematic errors, and it is necessary to artificially modify the semi-empirical $\log \mathrm{gf}$ values of some of the blending lines in order to achieve a good fit to the observations. This procedure, which is discussed in detail in Leckrone (1981) for example, is bounded by the requirement that the adopted mix of $\log$ gf values for the blending lines must work for all of the stars we analyse. As long as the primary line being synthesized (in this case FeII $\lambda 2607$ ) is the dominate member of the blend, rather large errors in the calculated strengths and positions of the blending contaminates can be incurred without seriously affecting the derived abundance. Errors will typically be less than $0.1 \mathrm{dex}$, even if we ignore a major blending contributor. In 
the computation illustrated in Figure l, the log gf value used for the FeII resonance line is taken from a new critical compilation by Martin et al. (1986). Its uncertainty of approximately $10 \%$ is substantially lower than that of any currently available $\log$ gf values for FeII lines in the optical region. The lower part of Figure 1 shows this synthetic spectrum, convolved with the slit broadening function of the IUE spectrograph and rotated up to $12 \mathrm{~km} / \mathrm{s}$, compared to the line as observed with the IUE. The fit is reasonably good for an iron abundance $\log [\mathrm{N}(\mathrm{Fe}) / \mathrm{N}(\mathrm{H})]=-4 \cdot 17$.

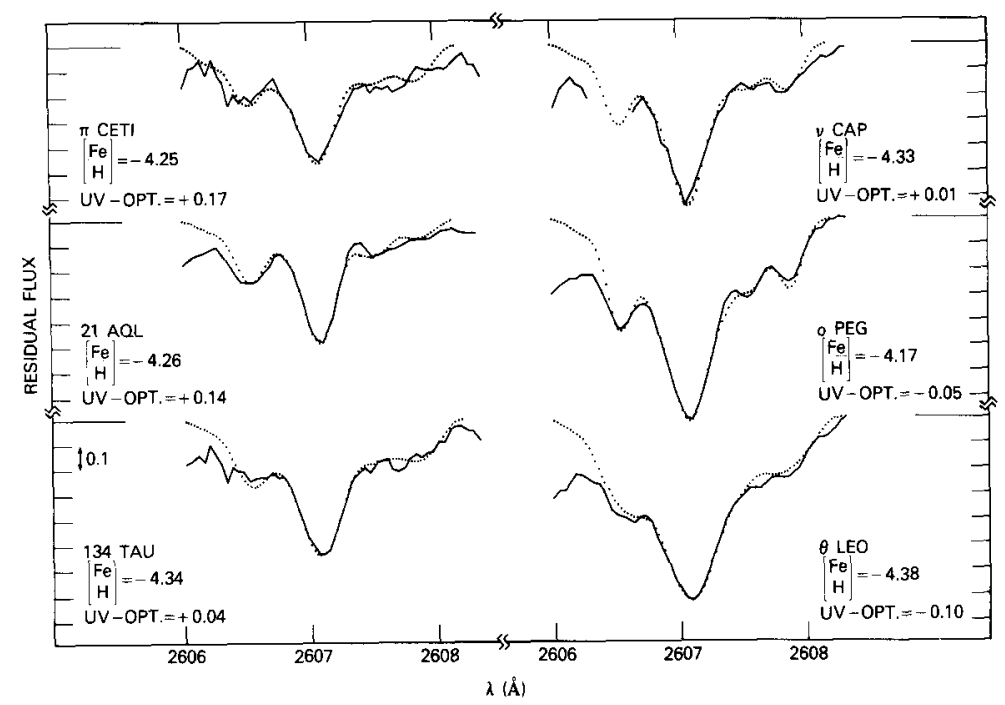

Figure 2. Spectral syntheses of FeII $\lambda 2607$ in six normal stars. Solid curves are features observed with IUE; dotted curves are suitably broadened theoretical profiles.

Figure 2 illustrates the syntheses of FeII(1) $\lambda 2607$ for the six normal stars included in Adelman and Leckrone's program. Using the procedures illustrated in detail in Figure 1 , one obtains a good fit to all the observations. The preliminary abundances derived from this single ultraviolet resonance line of FeII are compared to the iron abundances derived from optical region observations of the same stars in Table 2 . The generally good agreement between optical and ultraviolet values in the normal stars (top section of Table 2) is encouraging and gives us confidence to proceed with the analyses of numerous ultroviolet lines. There does appear to be a small systematic effect, which we do not yet understand, in that the optical abundances for the hotter stars tend to be lower than those derived from this single UV line. It will be of interest to see if this effect persists as we analyse additional $\mathrm{Fe}$ lines and as we continue to refine our data analysis techniques (for example, in the way we locate the continuum). We intend to complete 
similar analyses for about twelve additional ultraviolet FeII lines which have $\log$ gf values of quality $B$ or $B+$ on the U.S. National Bureau of Standards scale of accuracy, i.e. with uncertainties around $10 \%$. In addition we have good IUE data for about 25 other FeII lines, whose transition probabilities are less well known, for which astrophysical fvalues could be derived. We are interested not only in accurate absolute abundances, but, for the normal stars, in the possible variations in abundances from star-to-star. After iron we will proceed to $\mathrm{Mn}$, Co, $\mathrm{Ni}$ and other elements of the iron peak and ultimately to as much of the periodic table as possible.

TABLE 2

PRELIMINARY IRON ABUNDANCE RESULTS

$\begin{array}{rccc}\frac{\text { Star }}{\pi \text { Cet }} & \frac{\text { Optical }}{-4.42} & \frac{\lambda 2607}{-4.25} & \frac{\text { UV - Optical }}{+0.17} \\ 21 \text { Aq } 1 & -4.40 & -4.26 & +0.14 \\ 134 \text { Tau } & -4.38 & -4.34 & +0.04 \\ \nu \text { Cap } & -4.34 & -4.33 & +0.01 \\ \text { 0 Peg } & -4.12 & -4.17 & -0.05 \\ \theta \text { Leo } & -\frac{-4.28}{32 \pm 0.11} & -4 . \frac{-4.38}{29 \pm 0.08} & \frac{-0.10}{\sigma=0.11} \\ \text { Average } & -4.11 & \\ \text { Sun } & -4.37 & & \\ \text { I CrB } & -4.20 & -4.05 & +0.15 \\ \text { K Cnc } & -4.47 & -4.10 & +0.37\end{array}$

\section{PROSPECTS FOR RESEARCH WITH THE HUBBLE SPACE TELESCOPE}

It is evident from the work done by numerous people on the IUE spectra of $\mathrm{CP}$ and related normal stars that a rich store of information about the nature and origin of chemical anomalies resides in the complex ultraviolet spectra of these stars. The best tool for accurate quantitative studies of this region will undoubtedly be the High

Resolution Spectrograph (HRS) on the Hubble Space Telescope (HST), to be launched by NASA's Space Shuttle during the latter half of 1986. The HST will be maintained and repaired in orbit by Shuttle crew members over its lifetime of at least fifteen years. Its instruments can be replaced with even more sophisticated devices as time goes on. Recently several candidate second generation HST instruments were selected for study, including an echelle spectrograph with a two-dimensional, photoncounting imaging detector (the STIS or Space Telescope Imaging Spectrograph), which will allow simultaneous observations over a much wider wavelength interval than that covered by the HRS. Both the HRS and the STIS will have substantially wider dynamic ranges and lower levels of background noise than does the IUE. 
In addition to spectroscopy the HST will provide other important capabilities for the study of $\mathrm{CP}$ stars. The basic characteristics of the scientific instruments which will be on board the HST at launch are summarized in Table 3. The High Speed Photometer (HSP), which is capable of accurate photometry in time bins as short as $10 \mu \mathrm{sec}$, might well be. applied to the study of rapid variability throughout the ultraviolet and optical bands. The search for $\mathrm{CP}$ stars in clusters could be simplified by the high angular resolution ( 0.1 arcseconds) and the large selection of $\mathrm{UV}$ and optical filters in the two cameras. With regard to the HRS it is important to note that the highest resolving power $(\lambda / \Delta \lambda \simeq 95000)$ is obtained with an echelle grating which introduces a significant amount of scattered light. The scattered light background can be measured and subtracted out but at the expense of longer exposure times. On the other hand the intermediate 25000 resolving power, obtained in first order, offers almost twice the resolution of the IUE and a superbly dark background. Thus one must exercise judgement as to whether the highest possible signal-to-noise ratio or the highest possible resolution is needed for a given observation. Note that the resolving power in the 25000 mode might be improved to about 50000 by deconvolving the instrumental broadening function from data with a sufficiently high signal-to-noise. The detectors in the HRS are linear diode arrays (Digicons). This limits the width of the spectral interval that can be measured in a single exposure to about 5 to $18 \AA$ in the 95000 mode and to about 26 to $48 \AA$ in the 25000 mode.

TABLE 3

\section{HUBBLE SPACE TELESCOPE SCIENTIFIC INSTRUMENTS}

\begin{tabular}{|c|c|c|c|c|c|c|}
\hline & $\begin{array}{l}\text { WAVELENGTH } \\
\text { RANGE } \\
\text { (A) }\end{array}$ & $\begin{array}{l}\text { FIELD OF } \\
\text { VIEW } \\
\text { (ARCSEC) }\end{array}$ & $\begin{array}{l}\text { ANGULAA } \\
\text { RESOLUTION } \\
\text { (ARCSEC) }\end{array}$ & $\begin{array}{l}\text { SPECTRAL } \\
\text { RESOLVING } \\
\text { POWER }\end{array}$ & $\begin{array}{l}\text { MINIMUM } \\
\text { ACCUMULATION } \\
\text { TIME } \\
\text { (MSEC) }\end{array}$ & $\begin{array}{c}\text { "UIMITING } \\
\text { MAGNITUDE" } \\
\text { (mV' }\end{array}$ \\
\hline $\begin{array}{l}\text { WIDE FIELD AND } \\
\text { PLANETARY CAMERA }\end{array}$ & $1150-11500$ & $70-160$ & $0.1-0.2$ & FILTERS & 100 & 28 \\
\hline $\begin{array}{l}\text { FAINT OBJECT } \\
\text { CAMERA }\end{array}$ & $1150-7000$ & $<44$ & $<0.1$ & FHLTERS & 50 & 28 \\
\hline $\begin{array}{l}\text { HIGH RESOLUTION } \\
\text { SPECTROGRAPH }\end{array}$ & $1100-3200$ & $0.25-2.0$ & N/A & $\begin{array}{c}95000 \\
25000 \\
2500\end{array}$ & 200 & $12-16$ \\
\hline $\begin{array}{l}\text { FAINT OBJECT } \\
\text { SPECTROGRAPH }\end{array}$ & $1150-8000$ & 0.14 .0 & N/A & $\begin{array}{c}1200 \\
200\end{array}$ & 10 & $23-26$ \\
\hline $\begin{array}{l}\text { HIGH SPEED } \\
\text { PHOTOMETER }\end{array}$ & $1150-9000$ & $0.4-1.0$ & N/A & FILTERS & 0.01 & 24 \\
\hline $\begin{array}{l}\text { FINE GUIDANCE } \\
\text { SENSOR ASTROMETER }\end{array}$ & $4670-7000$ & $\approx 500$ & 0.002 & FILTERS & N/A & 19 \\
\hline
\end{tabular}

In the following discussion I use the example of the HgII resonance line at $1942 \AA$, which was the subject of my recent paper (Leckrone 1984), to illustrate the potential applications of the HRS to the study of the 


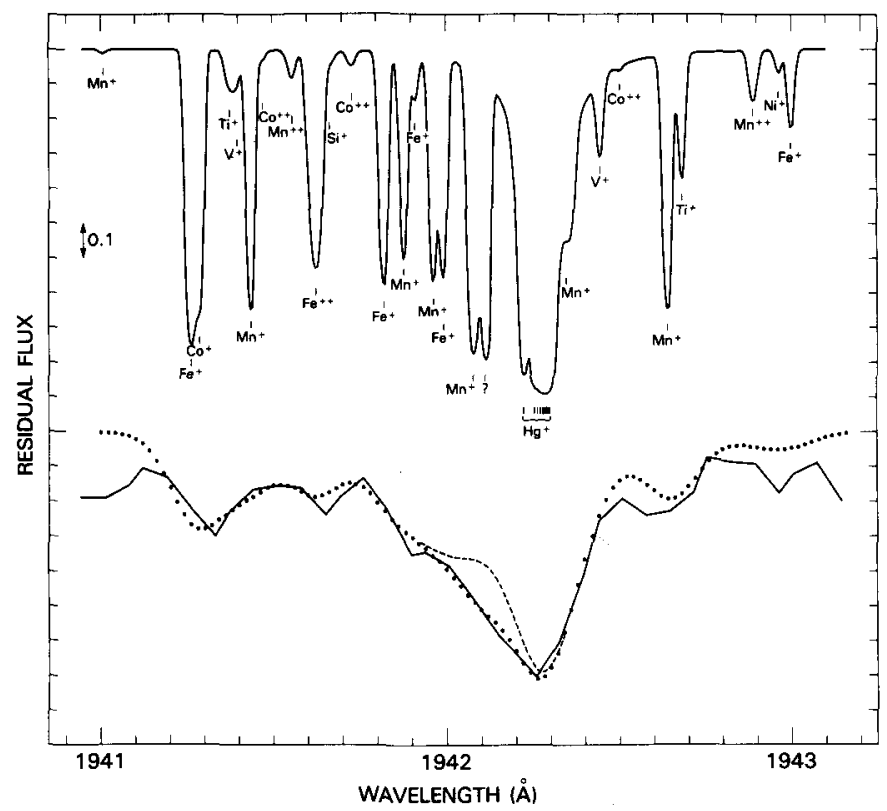

Figure 3. Spectral synthesis of the HgII $\lambda 1942$ resonance line in $1 \mathrm{CrB}$. Top - theoretical spectrum with $\log [\mathrm{N}(\mathrm{Hg}) / \mathrm{N}(\mathrm{H})]=-6.1$. Bottom IUE observation (solid curve) compared to suitably broadened theoretical computation (dots) and to a theoretical computation from which an unidentified blending feature (question mark in upper figure) has been omitted (dashed curve).

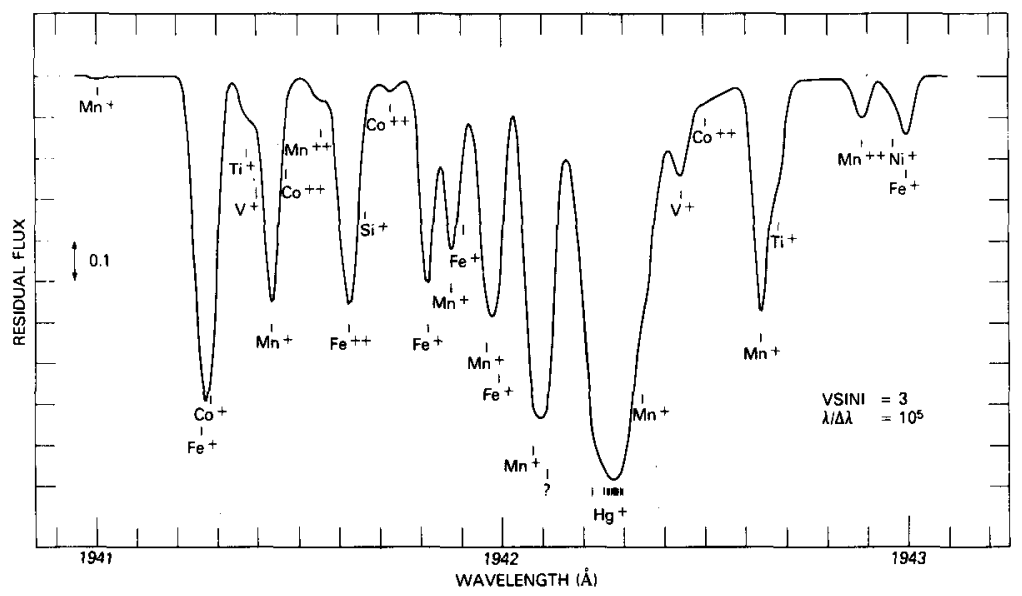

Figure 4. Theoretical spectrum from Figure 3 broadened with the expected instrumental function for the highest resolution mode of the High Resolution Spectrograph. 
ultraviolet spectra of the sharpest-lined stars, many of which are included in the various classes of chemically peculiar stars. Why is one interested in the $\mathrm{HgII}$ resonance lines? Prior to the IUE the $\mathrm{Hg}$ anomaly rested entirely on observations of $\mathrm{HgII} \lambda 3984$, p1us the very weak $\mathrm{HgI}$ $\lambda 4358$ line seen in only a few stars. Figure 9 of Leckrone (1984) shows computed curves of growth for $\lambda 3984$ as a function of isotopic mix parameter, $q$, effective temperature and microturbulent velocity, $v_{t}$. It is clear that the strength of $\lambda 3984$ is extremely sensitive to the choice of $\mathrm{q}$ and $\mathrm{v}_{\mathrm{t}}$. Small errors in these parameters will produce large errors in the $\mathrm{Hg}$ abundance derived from this line. This is the cause of much of the large star-to-star scatter in $\mathrm{Hg}$ abundances found in the literature. In contrast, as is shown in Figure 3 of Leckrone (1984), the resonance line of HgII at $1942.275 \AA$ is insensitive to all three parameters and is only slightly sensitive to the adopted value of the damping constant (it is dominated by radiation damping, which is easily calculated). Thus, this line should yield accurate abundances, provided the problems of line blending discussed previously can be overcome. The detailed synthesis of $\lambda 1942$ in IUE observations of the HgMn star l CrB is shown in Figure 3. The technique used is identical to that discussed earlier for the FeII lines. That is the Kurucz-Peytremann log gf values of the contaminate blending lines had to be adjusted to achieve an acceptable fit to the observations in all the stars considered. Also in this case an extra blending line of unknown identity ( $\mathrm{I}$ assumed TiII) had to be added to the blend to explain the asymmetry of the line core on its short wavelength side. This is consistent with the findings of Cowley and Johannson (in this colloquium) that there are a significant number of missing lines in the available line lists. At the bottom of Figure 3 is this same synthetic spectrum, convolved with rotational and IUE instrumental broadening functions, and overlaid on the observed feature in $\mathrm{l} \mathrm{CrB}$. The dashed line shows the theoretical spectrum without the addition of the unknown blending contaminate, for comparison. Figure 4 illustrates the synthetic spectrum from Figure 3 convolved with the $3 \mathrm{~km} / \mathrm{s}$ rotational broadening function and with the instrumental broadening function of the HRS in the 95000 resolving power mode. Most of the blending lines, whose identities we could only guess in the IUE spectra are now resolved, and even some of the weak features show up as partially resolved bumps or shoulders on the stronger lines. In addition we are more likely to accurately locate the line-free continuum in such high resolution observations (this is a difficult but not insurmountable problem in the IUE spectra). The signal-to-noise ratio in HRS observations of this sort will be limited only by the length of time one chooses to devote to counting photons, but in a realistic situation would likely be at least a factor of two better than in the best coadded IUE spectra. The problem then becomes one of adequately supporting the atomic physicists who are interested in providing the accurate atomic data needed to do justice to such observations.

In Figure $5 \mathrm{I}$ have focused on an approximately $1 \AA$ spectral interval around $\lambda 1942.275$ and have performed the computation of the synthetic spectrum for two extreme values of the isotopic mix parameter, $q$. The 
value $q=-0.1$ corresponds to a nearly terrestrial mix (as in the HgMn star $\mu$ Lep, for example), while the value ${ }_{04} q=+3.0$ corresponds to an extreme isotopic anomaly (almost pure $\mathrm{Hg}^{204}$ ) as is found in $\mathrm{X}$ Lup. At this resolving power and with good signal-to-noise one will be able to discriminate to some degree the isotopic structure of this $\mathrm{Hg}$ line to compare it to previously reported results for $\lambda 3984$.

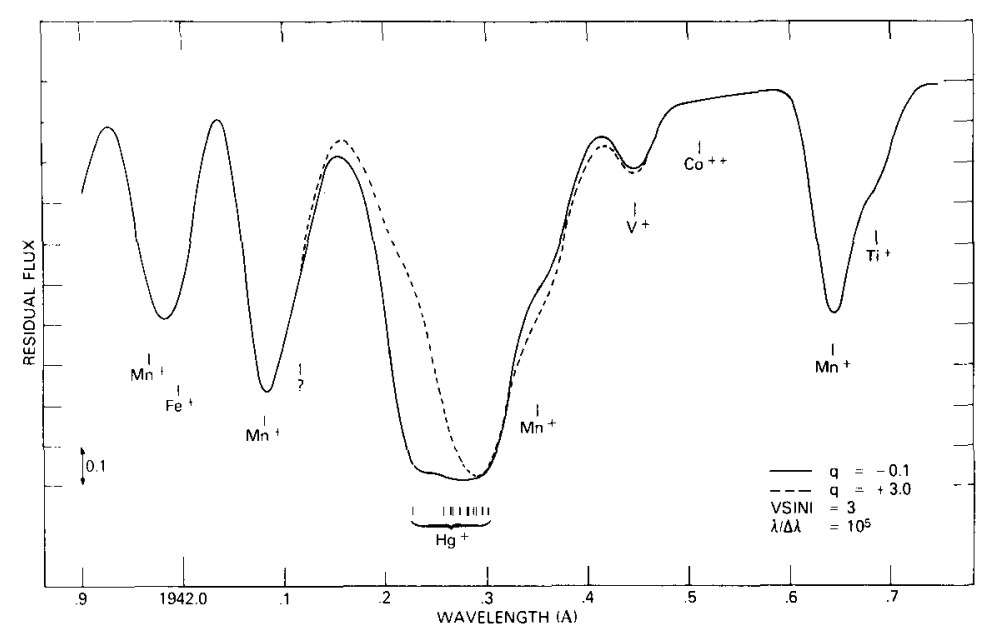

Figure 5. Theoretical computations of HgII $\lambda 1942$ for two extreme values of the isotopic mix parameter, $q$, broadened to $v$ sin $i=3 \mathrm{~km} / \mathrm{s}$ and degraded to the highest resolution of the HRS.

\section{EARLY OBSERVATIONS OF CP STARS WITH THE HST}

The first observations of $\mathrm{CP}$ and related normal stars with the HST will be those I carry out as a member of the High Resolution Spectrograph Team. My program is made up of eight separate observational objectives, which focus on non-magnetic HgMn stars, a field horizontal branch star, and normal sharp-lined $B$ and $A$ main-sequence stars. The selection of objectives reflects my own judgement as to what scientific questions will be most amenable to immediate attack. with the new observational capabilities of the HRS, as well as my own historical research interests. Certainly the magnetic CP stars, the Am stars, the stars with anomalous He lines, etc., will also provide fruitful research opportunities with the HRS and the other HST instruments. In the following discussion, I summarize my observational programs both to give an idea of the types of research one might undertake with the HRS and to point out the opportunity that is now being provided to astronomers from all over the world to submit proposals to observe with the HST. My programs are constrained to about 30 hours of observing time spread out over 2.5 years - sufficient only to scratch the surface of the important research to be done. The data obtained will be protected for one year 
after the completion of the observations, after which time they will become part of the archives of HST data accessible to the general community.

The eight major parts of my observing program are listed below. Table 4 lists the target stars associated with these objectives.

Part 1. - Survey BII $\lambda 1362$ resonance line at $\lambda / \Delta \lambda \simeq 95000$ in $\mathrm{HgMn}$ and normal stars to establish $B$ abundance trends.

Part 2. - Establish Hg abundance in normal stars based on observations of resonance lines at $\lambda / \Delta \lambda \simeq 95000$.

Part 3. - Study the systematic behavior of UV lines of $\mathrm{HgI}$, $\mathrm{HgII}$ and $\mathrm{HgIII}$ and derive accurate $\mathrm{Hg}$ abundances in a wide range of $\mathrm{Hg}-\mathrm{rich}$ stars at $\lambda / \Delta \lambda \simeq 95000$.

Part 4. - Explore odd-even abundance patterns in HgMn stars (program constrained by time allocation to search for low-excitation lines of $\mathrm{Au}$ in stars known to be rich in $\mathrm{Pt}$ and $\mathrm{Hg}$ ) at $\lambda / \Delta \lambda \simeq 95000$.

Part 5. - Obtain complete UV spectra of selected HgMn stars with high signal-to-noise at $\lambda / \Delta \lambda \simeq 25000$ for line identification and abundance analyses.

Part 6. - Obtain complete UV spectra of selected normal B and A stars with high signal-to-noise at $\lambda / \Delta \lambda \simeq 25000$ to establish spectroscopic and abundance comparison standards.

Part 7. - Establish CNO abundances in a characteristic, bright field horizontal branch star using observations of selected low excitation lines at $\lambda / \Delta \lambda \simeq 25000$.

Part 8. - Obtain complete UV spectra of a bright sharp-lined normal A star and an extremely sharp-1ined HgMn star at $\lambda / \Delta \lambda \cong 95000$ (lower priority objective to be carried out if time permits).

TABLE 4

\section{HST PROGRAM STARS}

\begin{tabular}{|c|c|c|c|c|c|}
\hline Star (HD) & Name & Objective & Star (HD) & Name & Objective \\
\hline 1909 & $\overline{\mathrm{HR}} 89$ & 1,3 & 141556 & $\overrightarrow{x \text { Lup }}$ & $1,3,4,8$ \\
\hline 17081 & $\pi$ Cet & 2 & 143807 & I CrB & $1,3,5$ \\
\hline 27295 & 53 Tau & $1,3,4,5$ & 145389 & $\phi$ Her & 3,5 \\
\hline 33904 & $\mu$ Lep & 1,3 & 149121 & 28 Her & 3,4 \\
\hline 38899 & $134 \mathrm{Tau}$ & 2 & 174933 & $112 \mathrm{Her}$ & 1,3 \\
\hline 48915 & $\alpha \mathrm{CMa}$ & 8 & 182308 & HR 7361 & $1,3,4$ \\
\hline 78316 & $\times$ Cnc & $1,3,5$ & 190229 & HR 7664 & \\
\hline 89822 & HR 4072 & $1,3,4$ & 193432 & $\checkmark$ Cap & $1,2,4,6$ \\
\hline 97633 & $\theta$ Leo & 2 & 193452 & HR 7775 & $1,3,4$, \\
\hline 110073 & $\mathrm{HR}, 4817$ & 1,3 & 207857 & HR 8349 & 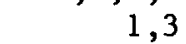 \\
\hline 129174 & $\pi^{1}$ Boo & 1,3 & 214994 & o Peg & 2,3, \\
\hline 130095 & & 7 & 215573 & $\xi$ Oct & $1,2,3,4$, \\
\hline
\end{tabular}

I strongly encourage my fellow CP-star aficionados to consider taking advantage of the new opportunities the HST will offer to help us resolve the mysteries of these enigmatic objects. 


\section{REFERENCES}

Adelman, S.J. and Leckrone, D.S. 1985, NASA IUE Newsletter, No. 28, in press.

Dworetsky,M.,M., Storey, P.J. and Jacobs, J.M. 1984, Physica Scripta, $\mathrm{T} 8, \mathrm{p} .39$.

Jacobs, J.M. and Dworetsky, M.M. 1981, in Upper Main Sequence Chemically Peculiar Stars, $23^{\text {rd }}$ Liege International Astrophysical Colloquium, p.153.

Jacobs, J.M. and Dworetsky, M.M. 1982, Nature, 299, p.535.

Kurucz, R.L. 1981, Smithsonian Astrophysical Obs. Special Report, No. 390 .

Kurucz, R.L. and Peytremann, E. 1975, Smithsonian Astrophysical Obs. Special Report, No. 362.

Leckrone, D.S. 1975, in Physics of Ap-Stars, IAU Coll. No. 32, (ed., W. Weiss, H. Jenkner and H. Wood), University of Vienna, p.465.

Leckrone, D.S. 1980, Highlights of Astr., 5, p.277.

Leckrone, D.S. 1981, Astrophys. J., 250, p.687.

Leckrone, D.S. 1984, Astrophys. J., 286, p.725.

Martin, G.A., Fuhr, J.R. and Wiese, W.L. 1986, in preparation.

Sadakane, K., Takada, M. and Jugaku, J. 1983, Astrophys. J., 274, p.261.

Sadakane, K., Jugaku, J. and Takada-Hidai, M. 1985, Astrophys. J., 297 , p. 240 . 